\title{
Diagnostic Accuracy of Markers of Insulin Resistance (HOMA-IR) and Insulin Sensitivity (QUICKI) in Gestational Diabetes
}

\author{
Athar lqbal Paracha, Zujaja Hina Haroon, Muhammad Aamir and Afshan Bibi \\ Department of Chemical Pathology and Endocrinology, Armed Forces Institute of Pathology (AFIP), Rawalpindi, Pakistan
}

\begin{abstract}
Objectives: To determine the diagnostic accuracy of HOMA-IR, and QUICKI in diagnosing gestational diabetes mellitus (GDM) considering oral glucose tolerance test (OGTT) as gold standard.

Study Design: Cross-sectional analytical study.

Place and Duration of Study: Department of Chemical Pathology, Armed Forces Institute of Pathology, Rawalpindi from September 2020 to February 2021.

Methodology: Pregnant women with gestational age between 24 to 28 weeks, who reported to Endocrine Clinic of AFIP for OGTT, were included in the study. OGTT was performed by following ADA guidelines. Sample for fasting insulin was collected along with first fasting sample of OGTT. HOMA-IR and QUICKI were calculated simultaneously. Percentage was used for qualitative variable while median (IQR 25th-75th) was applied for quantitative variables. OGTT was used as gold standard for calculation of diagnostic accuracy of HOMA-IR and QUICKI.

Results: Out of 182 patients, $74(40.6 \%)$ were found to have GDM on OGTT while $108(59.4 \%)$ had normal OGTT. Women with GDM $(n=74)$ had median values of fasting insulin 15.9 (IQR 11.2-17.77), HOMA-IR 3.5 (IQR 2.6-4.1) and QUICKI 0.31 (IQR 0.30-0.33) as compared to median values of fasting insulin 8.0 (IQR 5.9-10.3), HOMA-IR 1.60 (IQR 1.12-2.03) and QUICKI 0.35 (IQR 0.34-0.37) in patients $(n=108)$ with normal response to OGTT, $(p<0.001)$. On logistic regression analysis, there was a strong association of HOMA-IR and QUICKI with gestational diabetes mellitus ( $p<0.001$, accuracy $84.6 \%$ ). HOMA-IR at cutoff of $\leq 2$ had $94.5 \%$ sensitivity, $72.2 \%$ specificity, $70 \%$ PPV, 95.1\% NPV, and $81.31 \%$ overall diagnostic accuracy with 0.913 AUC. QUICKI at cutoff of 0.34 had $86.4 \%$ sensitivity, $83.3 \%$ specificity, $78.0 \%$ PPV, $90.0 \%$ NPV, and $84.61 \%$ overall diagnostic accuracy with 0.905 AUC.

Conclusion: Fasting insulin and HOMA-IR were significantly higher while QUICKI was lower in patients of GDM as compared to non-GDM pregnant patients at 24 to 28 weeks of gestation. Being more convenient for patients, it has the potential to be used as screening tool for gestational diabetes.
\end{abstract}

Key Words: HOMA IR, QUICKI, Gestational diabetes mellitus, Insulin.

How to cite this article: Paracha Al, Haroon ZH, Aamir M, Bibi A. Diagnostic Accuracy of Markers of Insulin Resistance (HOMA-IR) and Insulin Sensitivity (QUICKI) in Gestational Diabetes. J Coll Physicians Surg Pak 2021; 31(09):1015-1019.

\section{INTRODUCTION}

Gestational diabetes mellitus (GDM) is a condition of glucose intolerance that is first recognised in pregnancy. American Diabetes Association (ADA) has defined GDM as the diabetes, which is diagnosed during the second and third trimesters of pregnancy. ${ }^{1}$

Correspondence to: Dr. Athar Iqbal Paracha, Department of Chemical Pathology and Endocrinology, Armed Forces Institute of Pathology, Rawalpindi, Pakistan

E-mail: athar_paracha@hotmail.com

Received: May 21, 2021; Revised: August 09, 2021;

Accepted: August 28, 2021

DOI: https://doi.org/10.29271/jcpsp.2021.09.1015
It affects about one out of every six pregnancies globally. ${ }^{2}$ Its prevalence has increased by more than $30 \%$ in the past few years in many developing countries, leading to an emerging worldwide epidemic. ${ }^{3}$ In Pakistan, different studies have reported prevalence of GDM to be around $8 \% .{ }^{4}$

The main pathogenesis behind development of GDM is because of the hormones such as cortisol, estrogen and human placental lactogen. These hormones are released from placenta during pregnancy and they mediate insulin resistance. This insulin resistance progressively increases during second trimester; and in women who are unable to produce sufficient insulin to cater for this insulin resistance, develops GDM. Therefore, screening for GDM is recommended in 24 to 28 weeks of gestation. ${ }^{5}$

According to the ADA 2019 criteria, the diagnosis of GDM in patients without any pre-existing diabetes can be made by 
performing oral glucose tolerance test (OGTT) with 75 gram glucose after overnight fasting of more than eight hours. Following one step procedure, a total of three plasma glucose samples are required which include fasting sample, post-glucoseload1-hour and 2-hoursample. ${ }^{6}$

However, OGTT although having high potency for diagnosis of GDM has some limitations which include prior appointments, prolong duration, taking oral glucose which may cause nausea and vomiting leading to termination of the test and multiple pricks forperforming the procedure. ${ }^{7}$

Homeostasis model assessment-estimated insulin resistance (HOMA-IR) which was introduced in 1985 is currently being widely used for determination of insulin resistance. ${ }^{8}$ The quantitative insulin-sensitivity check index (QUICKI) is another accurate marker for estimation of sensitivity of insulin. ${ }^{9}$ Both these markers are single prick tests, which require patients to be in fasting state and can be calculated from fasting sample of plasma glucose and serum insulin. The main reason for development of GDM is insulin resistance. ${ }^{10}$

The rationale of this study was to determine whether these biomarkers of insulin resistance (HOMA-IR) and insulin sensitivity (QUICKI) can be used as a diagnostic tool for gestational diabetes mellitus as these biomarkers are more convenient for patients and requires only a single fasting sample.

The study objective was to determine diagnostic accuracy of markers of insulin resistance estimated by HOMA-IR and insulin sensitivity estimated by QUICKI in gestational diabetes mellitus, considering OGTT following guidelines of American Diabetes Association as gold standard.

\section{METHODOLOGY}

A cross-sectional study was conducted at Department of Chemical Pathology, Armed Forces Institute of Pathology, Rawalpindi, over a duration of six months from September 2020 to February 2021 afterapproval from the Institutional Ethical Committee.

Pregnant female patients with gestational age between 24 to 28 weeks, who reported to Endocrine Clinic of AFIP for OGTT, were included in this study. Informed written consent was taken from the patients. Exclusion criteria were patients, who had fasting of less than eight hours, diagnosed with GDM in previous pregnancies or had multiple comorbid condition, like renal impairment, cardiovascular disease or twin pregnancy.

OGTT was performed by collecting fasting blood sample between $0800 \mathrm{hrs}-0900 \mathrm{hrs}$. Venous blood sample ( $3 \mathrm{ml}$ ) was collected in sodium fluoride tube for plasma glucose. Fasting insulin sample ( $2 \mathrm{ml}$ ) was collected simultaneously in gel tube. To the patients, 75 grams glucose dissolved in $200 \mathrm{ml}$ of water were given. Venous blood samples for glucose estimation were collected after one and two hours of glucose administration.

Samples centrifugation was done at $3500 \mathrm{rpm}$ for three minutes and was analysed within two hours of collection. Plasma glucose was analysed on ADVIA 1800 chemistry auto analyser by hexokinase method. While serum insulin levels were analysed on ADVIA
Centaur XP auto analyser by chemiluminescense method, three levels of controls including low, normal, and high were run with each batch of plasma glucose and serum insulin as a part of internal quality control.

Gestational diabetes mellitus was labelled on OGTT, following one-step strategy of American Diabetes Association 2020 guidelines. Cutoff for plasma glucose were plasma glucose (Fasting) $<5.1 \mathrm{mmol} /$, post-glucose load (1 hour sample) $<10 \mathrm{mmol} / \mathrm{l}$ and post-glucose load (2-hour sample) $<8.5 \mathrm{mmol} / \mathrm{l}$. Patients with any one value falling above the cutoff were labelled as GDM.

HOMA-IR was determined by using the equation, HOMA-IR = (fasting glucose $x$ fasting insulin)/ 22.5. ${ }^{11}$ Patients with HOMA IR $>2$ were considered to have insulin resistance. ${ }^{12}$ Whereas, QUICKI was estimated by using the equation, QUICKI $=[1 /(\log$ fasting Insulin + log fasting glucose)]. ${ }^{13}$ Patients with QUICKI < 0.34 were considered to have Insulin resistance.

Statistical analysis was done by using SPSS version 26 . For determination of normality of data, Shapiro-Wilk test was applied which revealed that the data had non-parametric distribution. Percentage was used for qualitative variables while median with IQR $25^{\text {th }}-75^{\text {th }}$ percentile was used for quantitative variables such as fasting insulin, HOMA-IR and QUICKI. Median comparison of HOMA-IR, QUICKI and fasting insulin was done by Mann-Whitney U-test, $p<0.05$ was considered to be statistically significant. Logistic regression analysis was used to determineassociation of HOMA-IR, QUICKI and fasting insulin with GDM. In each patient, OGTT $(75 \mathrm{~g})$ was considered as gold standard for diagnosing gestational diabetes mellitus and for the determination of the sensitivity, specificity, PPV, NPV and diagnostic accuracy of HOMA-IR and QUICKI.

\section{RESULTS}

A total of 182 pregnant women, having mean age of $29.47 \pm$ 4.03 years, were included in the study. Out of those, $74(40.6 \%)$ were found to have GDM on OGTT while 108 (59.4\%) were found to be negative for GDM. Women with GDM $(n=74)$ had median values of fasting insulin 15.9 (IQR 11.2-17.77), HOMA-IR 3.5 (IQR 2.6-4.1) and QUICKI 0.31 (IQR 0.30-0.33). Whereas, women who did not have GDM $(n=108)$ had median values of fasting insulin 8.0 (IQR 5.9-10.3), HOMA-IR 1.60 (IQR 1.12-2.03) and QUICKI 0.35 (IQR 0.34-0.37). Median fasting insulin, HOMAIR was high and QUICKI was low in patients who had GDM as compared to those who did not have GDM. The difference between medians of these quantitative variables when compared by using Mann-Whitney U-test was found to be statistically significant with $p<0.001$. On logistic regression analysis, there was strong association of HOMA-IR and QUICKI with gestational diabetes mellitus having $p<0.001$ and accuracy of $84.6 \%$.

In women, who had GDM ( $n=74)$, 70 had HOMA IR value of $>2$ while only four women had HOMA-IR $\leq 2$. While in those, who didn't have GDM ( $n=108), 78$ women had HOMA-IR $\leq 2$ and 30 women had HOMA-IR $>2$. A total of 100 women (54.9\%) had HOMA-IR $>2$, while 82 women (45.05\%) had HOMA-IR $\leq 2$. At cut 
off of $\leq 2$, the odds ratio, sensitivity, specificity, positive predictive value and negative predictive value of HOMA-IR for determining GDM were 9.75 (Cl2.39-39.67), 94.5\%, 72.2\%, 70\% and $95.1 \%$, respectively. Diagnostic accuracy was $81.31 \%$ (Table I).

Table I: Diagnostic accuracy of HOMA-IR in detection of GDM in pregnant patients with gestational age of 24-28 weeks taking OGTT as gold stan$\operatorname{dard}(n=182)$.

\begin{tabular}{|l|c|c|c|}
\hline & GDM & Non-GDM & Total \\
\hline HOMA IR $>2$ & 70 & 30 & 100 \\
\hline HOMA IR $\leq 2$ & 4 & 78 & 82 \\
\hline Total & 74 & 108 & 182 \\
\hline Sensitivity & $70 /(70+4) * 100=94.6 \%$ \\
\hline Specificity & $78 /(78+30) * 100=72.2 \%$ \\
\hline Positive predictive value & $70 /(70+30) * 100=70 \%$ \\
\hline Negative predictive value & $78 /(4+78) * 100=95.1 \%$ \\
\hline Diagnostic accuracy & $70+78 /(70+4+30+78) * 100=81.31 \%$ \\
\hline
\end{tabular}

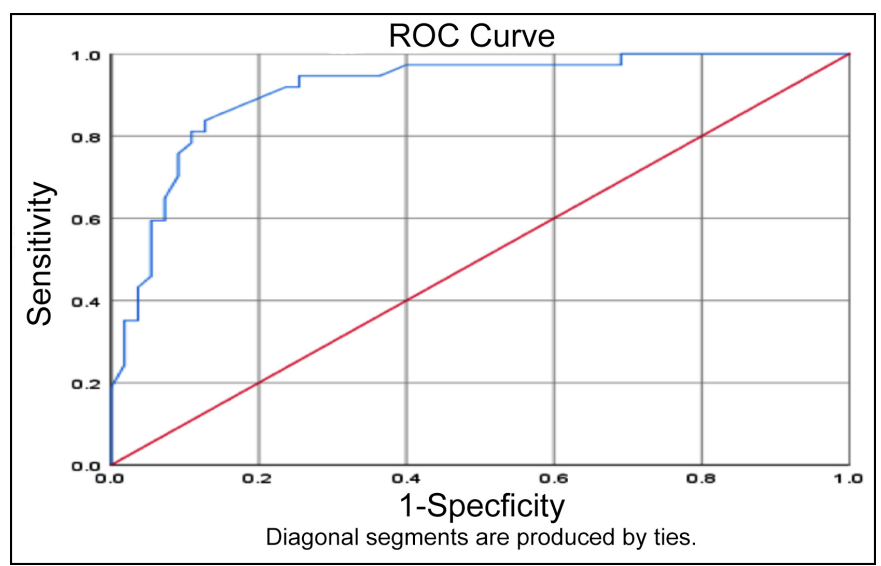

Figure 1: ROC-AUC analysis of HOMA-IR.

On ROC curve of HOMA-IR, the best cutoff point was 2.035 with sensitivity $94.6 \%$ and specificity $74.5 \%$. AUC for HOMA-IR was 0.913 as shown in Figure 1 .

In women who had GDM ( $n=74), 64$ had QUICKI $<0.34$, while 10 had QUICKI $\geq 0.34$. While in those, who didn't have GDM $(n=108), 90$ had QUICKI $\geq 0.34$ and 18 had QUICKI $<0.34$. A total of $82(45.05 \%)$ had QUICKI $<0.34$ while 100 (54.94\%) had $\mathrm{QUICKI} \geq 0.34$. At cutoff of $<0.34$, the odds ratio, sensitivity, specificity, positive predictive value, and negative predictive value of QUICKI was 7.11 (Cl 2.34-21.59), 86.4\%, 83.3\%, 78\% and $90 \%$, respectively. While diagnostic accuracy was $84.61 \%$ (Tablell).

Table II: Diagnostic accuracy of QUICKI in detection of GDM in pregnant patients with gestational age of 24-28 weeks taking OGTT as gold standard $(n=182)$.

\begin{tabular}{|l|l|l|l|}
\hline & GDM & Non-GDM & Total \\
\hline QUICKI $<0.34$ & 64 & 18 & 82 \\
\hline QUICKI $\geq 0.34$ & 10 & 90 & 100 \\
\hline Total & 74 & 108 & 182 \\
\hline Sensitivity & $64 /(64+10) * 100=86.4 \%$ \\
\hline Specificity & $90 /(90+18) * 100=83.3 \%$ \\
\hline Positive predictive value & $64 /(64+18) * 100=78.0 \%$ \\
\hline Negative predictive value & $90 /(90+10) * 100=90.0 \%$ \\
\hline Diagnostic accuracy & $64+90 /(64+10+18+90) * 100=84.61 \%$ \\
\hline
\end{tabular}

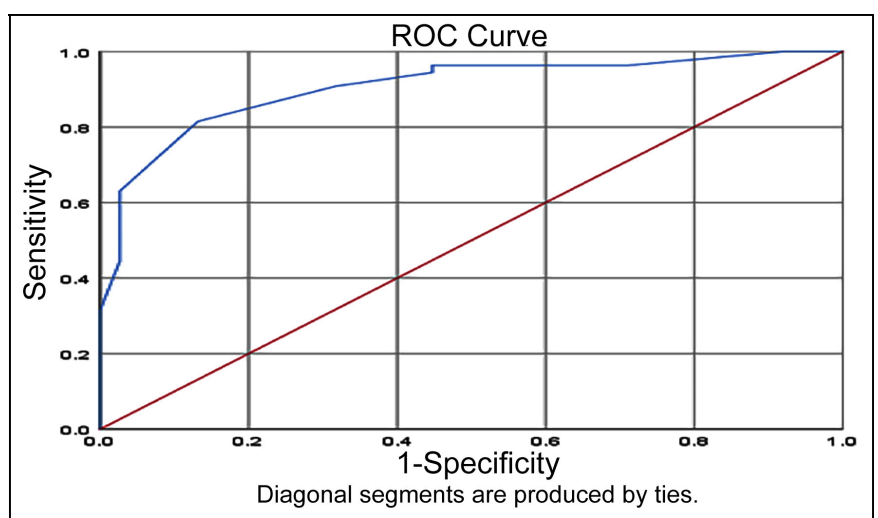

Figure 2: ROC-AUC analysis of QUICK.

On ROC curve of QUICKI, the best cutoff point was 0.325 with sensitivity $90.7 \%$ and specificity $68.4 \%$. AUC for QUICKI was 0.905, as shown in Figure 2 .

\section{DISCUSSION}

Pregnancy is a physiological hyperglycemic condition, resulting in progressive insulin resistance from mid-gestation. Insulin resistance is caused by release of diabetogenic hormones from placenta during pregnancy. These hormones include placental ACTH, human placental lactogen, placental growth hormone, estrogen and progesterone..$^{14}$ The hyperglycemia upto a certain level is essential for developmentoffetus. However, whensecretion of insulin from pancreas is inadequate to cater for the insulin resistance, patients develop gestational diabetes. ${ }^{15}$

The phenomena of insulin resistance was also evident in the present study where a statistically significant difference was demonstrated in fasting insulin, HOMA-IR and QUICKI values between GDM and non-GDM pregnant patients. The present study results were in consonance with other similar studies. Adam et al, also illustrated a significant difference in fasting insulin, HOMA-IR and QUICKI. In their study, the patients having GDM had mean fasting insulin $20 \mathrm{ulU} / \mathrm{ml}$, HOMA-IR 2.67 and QUICKI 0.636, while non-GDM pregnant patients had mean fasting insulin $11 \mathrm{ulU} / \mathrm{ml}$, HOMA-IR 1.26 and QUICKI 0.746. ${ }^{14}$ Endo et al. studied the difference in insulin sensitivity in all three trimesters and concluded that HOMA-IR in patients with GDM was significantly $(p<0.01)$ higher than those with normal-weight non-GDM pregnant patients. In patients with GDM, HOMA-IR significantly increased $(p<0.05)$ with advancement in pregnancy. Whereas, QUICKI was also significantly $(p<0.01)$ lower in patients having GDM as compared to normal-weight non-GDM patients and there was a significant decrease $(p<0.05)$ in QUICKI with progression of pregnancy. ${ }^{16}$ Wang et al. also concluded that HOMA-IR was significantly higher in the later stage of pregnancy in women with GDM as compared to non-GDM women. ${ }^{17}$ As Insulin resistance is a distinctive feature for progression to GDM, hence these biomarkers have the potential to be utilised as a screening and diagnostic tool forGDM.

In this study, the sensitivity of HOMA-IR was found higher than QUICKI, while QUICKI was found to be more specific. The sensi- 
tivity of HOMA-IR at cutoff value of $\leq 2$ was $94.5 \%$ with negative predictive value of $95.1 \%$. The specificity of QUICKI at cutoff of $\geq 0.34$ was $83.3 \%$ as compared to $72.2 \%$ in HOMA-IR. Ozcimen et al. conducted a study on 271 patients in their first trimester of pregnancy and concluded that HOMA-IR at cutoff value of 2.60 had sensitivity of $100 \%$, specificity of $94 \%$ and overall diagnostic accuracy of $92 \%{ }^{18}$ Alptekin et al., in a study conducted in 250 primigravida patients in their first trimester, found that at cutoff value of 2.08 HOMA- IR had $90 \%$ sensitivity and $61 \%$ specificity. ${ }^{19}$

GDM, if left untreated or undiagnosed, may lead to adverse maternal-fetal outcome such as fetal macrosomia, hypoglycemia of neonate, premature delivery, increased cesarean delivery rate, pre-eclampsia, birth injuries and shoulder dystocia. ${ }^{20}$

OGTT is used as a gold-standard test for the diagnosing GDM. In low risk patients, screening for GDM is done between 24 to 28 weeks of gestation. Drawback of this test is that it is quiet cumbersome, requires multiple sampling, time consuming; and unpleasant for some patients already having nausea. Thus, the scenario consequently leads to poor compliance by both the patients and healthcare professionals in achieving the target of maximum screening of GDM during pregnancy. An idealscreening test must be acceptable, convenient, accessible, and affordable.

In this study, HOMA-IR at cutoff of $\leq 2$ had high sensitivity $(94.5 \%)$ and high negative predictive value (95.1\%). HOMA-IR being simpler, more convenient test, requiring only a single fasting sample which can be used as a screening biomarker for GDM. HOMA-IR which may help in achieving the target for maximum screening and taking control of this emerging global pandemic in a better way. However, HOMA-IR being less specific, $(72.2 \%)$ the authors recommend that patients with HOMA-IR > 2 should undergo OGTT for confirmation of GDM.

The main limitation of this study was that it was single-centre study, conducted in pregnant patients with gestational age between 24 and 28 weeks. Secondly, as the insulin sensitivity varies amongst different races/ethnic groups, ${ }^{21}$ and the study was conducted only in Pakistani population. Further multicentre studies with larger sample size are recommended to determine cutoff of HOMA-IR and QUICKI at different stages of pregnancies.

\section{CONCLUSION}

Fasting insulin and HOMA-IR were significantly higher while QUICKI was lower in patients with GDM as compared to nonGDM pregnant patients at 24 to 28 weeks of gestation. HOMA-IR at cutoff of $\leq 2$ had a sensitivity of $94.5 \%$ and NPV of $95.1 \%$, which was higher than QUICKI ( $86.4 \%$ sensitivity and $90 \% \mathrm{NPV}$ ). These biomarkers determine the severity of insulin resistance and can be used as a potential screening tool for GDM in future. However, further studies are required to determine cutoff at different trimesters of pregnancy and in different ethnic groups.

\section{ETHICALAPPROVAL:}

Ethical approval of the study was given by the Institutional Review Board of AFIP prior to the launch of the study.

\section{PATIENTS' CONSENT:}

The informed written consent of each subject was obtained for study and publishing purposes.

\section{CONFLICT OF INTEREST:}

The authors declared no conflict of interest.

\section{AUTHORS' CONTRIBUTION:}

AIP: Data collection, data analysis, results, discussion and literature review.

ZHH: Data analysis, results, discussion and literature review. MA: Results, discussion and literature review.

$A B$ : Discussion and literature review.

\section{REFERENCES}

1. American diabetes association classification and diagnosis of diabetes. Diabetes Care 2015; 38(Suppl 1):S8-S16. doi: 10.2337/dc15-S005.

2. Goyal A, Gupta Y, Singla R, Kalra S, Tandon N. American diabetes association "standards of medical care - 2020 for gestational diabetes mellitus": A critical appraisal. Diabetes Therapy 2020; 11(8):1639-44. . doi: 10.1007/s13300020-00865-3.

3. Zhu $Y$, Zhang $C$. Prevalence of gestational diabetes and risk of progression to type 2 diabetes: A global perspective. Current Diabetes Reports 2016; 16(1):7. doi: 10.1007/ s11892-015-0699-x.

4. Riaz M, Nawaz A, Masood SN, Fawwad A, Basit A, Shera AS. Frequency of gestational diabetes mellitus using DIPSI criteria, a study from Pakistan. Clin Epidemiol Global Health 2019; 7(2):218-21.

5. Rani PR, Begum J. Screening and diagnosis of gestational diabetes mellitus, where do we stand. J Clin Diagnostic Res 2016; 10(4):QE01. doi: 10.7860/JCDR/2016/17588.7689.

6. American diabetes association. 2. Classification and diagnosis of diabetes: Standards of medical care in diabetes-2019. Diabetes Care 2019; 42(Suppl 1):S13-S28.

7. Babaniamansour S, Aliniagerdroudbari E, Afrakhteh $M$, Hosseinpanah F, Farzaneh F, Niroomand M. Can fasting plasma glucose replace oral glucose-tolerance test for diagnosis of gestational diabetes mellitus. Diabetol Int 2021; 12(3):277-285. doi: 10.1007/s13340-020-00484-0.

8. Qu HQ, Li Q, Rentfro AR, Fisher-Hoch SP, McCormick JB. The definition of insulin resistance using HOMA-IR for Americans of Mexican descent using machine learning. Plos One 2011; 6(6):e21041. doi: 10.1371/journal.pone.0021041.

9. Chen H, Sullivan G, Quon MJ. Assessing the predictive accuracy of QUICKI as a surrogate index for insulin sensitivity using a calibration model. Diabetes 2005; 54(7):1914-25. doi: 10.2337/diabetes.54.7.1914.

10. Buchanan TA, Xiang A, Kjos SL, Watanabe R. What is gestational diabetes? Diabetes Care 2007; 30(Suppl 2):S105-11.

11. Matthews DR, Hosker JP, Rudenski AS, Naylor BA, Treacher DF, Turner RC. Homeostasis model assessment: insulin 
resistance and beta-cell function from fasting plasma glucose and insulin concentrations in man. Diabetologia 1985; 28(7):412-9. doi: 10.1007/BF00280883.

12. Salgado AL, Carvalho LD, Oliveira AC, Santos VN, Vieira JG, Parise ER. Insulin resistance index (HOMA-IR) in the differentiation of patients with non-alcoholic fatty liver disease and healthy individuals. Arquivos de gastroenterologia 2010; 47(2):165-9. doi: 10.1590/ s0004-28032010000200009.

13. Katz A, Nambi SS, Mather K, Baron AD, Follmann DA, Sullivan $G$, et al. Quantitative insulin sensitivity check index: A simple, accurate method for assessing insulin sensitivity in humans. J Clin Endocrinol Metab 2000; 85(7):2402-10. doi: 10.1210/jcem.85.7.6661.

14. Adam S, Pheiffer C, Dias S, Rheeder P. Association between gestational diabetes and biomarkers: A role in diagnosis. Biomarkers 2018; 23(4):386-91. doi: 10.1080/1354750X. 2018.1432690.

15. Kauffman RP, Castracane VD, Peghee D, Baker TE, Van Hook JW. Detection of gestational diabetes mellitus by homeostatic indices of insulin sensitivity: A preliminary study. Am J Obstetrics Gynecol 2006; 194(6):1576-82. doi: 10.1016/j.ajog.2006.01.010.

16. Endo S, Maeda K, Suto M, Kaji T, Morine M, Kinoshita T, et al. Differences in insulin sensitivity in pregnant women with overweight and gestational diabetes mellitus. Gynecol Endocrinol 2006; 22(6):343-9. doi: 10.1080/095135
90600724836.

17. Wang X, Yang T, Miao J, Liu H, Wu K, Guo J, et al. Correlation between maternal and fetal insulin resistance in pregnant women with gestational diabetes mellitus. Clin Lab 2018; 64(6):945-53. doi: 10.7754/Clin.Lab.2018. 171214.

18. Ozcimen EE, Uckuyu A, Ciftci FC, Yanik FF, Bakar C. Diagnosis of gestational diabetes mellitus by use of the homeostasis model assessment-insulin resistance index in the first trimester. Gynecological Endocrinol 2008; 24(4): 224-9. doi: 10.1080/09513590801948416.

19. Alptekin H, Çizmecioğlu A, Işık H, Cengiz T, Yildiz M, lyisoy MS. Predicting gestational diabetes mellitus during the first trimester using anthropometric measurements and HOMAIR. J Endocrinol Invest 2016; 39(5):577-83. doi: 10.1007/ s40618-015-0427-z.

20. Kumari R, Dalal V, Kachhawa G, Sahoo I, Khadgawat R, Mahey $R$, et al. Maternal and perinatal outcome in gestational diabetes mellitus in a tertiary care Hospital in Delhi. Indian J Endocrinol Metabolism 2018; 22(1):116. doi: 10.4103/ijem.IJEM_582_17.

21. Raygor V, Abbasi F, Lazzeroni LC, Kim S, Ingelsson E, Reaven GM, et al. Impact of race/ethnicity on insulin resistance and hypertriglyceridaemia. Diabetes Vascular Disease Res 2019; 16(2):153-9. doi: 10.1177/147916 4118813890. 